\title{
Review Article \\ Laser-Induced Damage Initiation and Growth of Optical Materials
}

\author{
Jingxia Yu, ${ }^{1}$ Xia Xiang, ${ }^{1}$ Shaobo He, ${ }^{2}$ Xiaodong Yuan, ${ }^{2}$ Wanguo Zheng, \\ Haibing $L \ddot{u},{ }^{2}$ and Xiaotao $\mathrm{Zu}^{1}$ \\ ${ }^{1}$ School of Physical Electronics, University of Electronic Science and Technology of China, Chengdu, 610054, China \\ ${ }^{2}$ Research Centre of Laser Fusion, China Academy of Engineering Physics, Mianyang, 621900, China \\ Correspondence should be addressed to Xiaotao Zu; xtzu@uestc.edu.cn
}

Received 28 February 2014; Accepted 3 June 2014; Published 16 July 2014

Academic Editor: Haiyan Xiao

Copyright (C) 2014 Jingxia Yu et al. This is an open access article distributed under the Creative Commons Attribution License, which permits unrestricted use, distribution, and reproduction in any medium, provided the original work is properly cited.

\begin{abstract}
The lifetime of optical components is determined by the combination of laser-induced damage initiation probability and damage propagation rate during subsequent laser shots. This paper reviews both theoretical and experimental investigations on laserinduced damage initiation and growth at the surface of optics. The damage mechanism is generally considered as thermal absorption and electron avalanche, which play dominant roles for the different laser pulse durations. The typical damage morphology in the surface of components observed in experiments is also closely related to the damage mechanism. The damage crater in thermal absorption process, which can be estimated by thermal diffusion model, is typical distortion, melting, and ablation debris often with an elevated rim caused by melted material flow and resolidification. However, damage initiated by electron avalanche is often accompanied by generation of plasma, crush, and fracture, which can be explained by thermal explosion model. Damage growth at rear surface of components is extremely severe which can be explained by several models, such as fireball growth, impact crater, brittle fracture, and electric field enhancement. All the physical effects are not independent but mutually coupling. Developing theoretical models of multiphysics coupling are an important trend for future theoretical research. Meanwhile, more attention should be paid to integrated analysis both in theory and experiment.
\end{abstract}

\section{Introduction}

When a low-intensity laser passes through a transparent material, little or no effect may be detected. However, when the intensity of the laser is increased, the effects of interaction between laser and material may become obvious, including temperature rise, expansion, strain, distortion, nonlinear absorption, and transmittance change. As the laser intensity increases further some nonreversible changes may occur in the materials or components, such as melting and evaporation, material softening and bending, pitting, cracking, and shattering.

Interaction between laser and optical components can cause permanent changes which are called laser-induced damage (LID) in the material. The laser-induced damage threshold (LIDT) is that level of radiation which initiates some alteration of the optics under examination. Damage sites and defects in the beam path cause a loss in transmitted energy to the target. Meanwhile, LID scatters light, which ablates or damages the structural materials in the vicinity of the optics. This in turn can contaminate the optics and initiate further damage [1-3]. Finally, damage growth beyond a critical size may cause catastrophic failure of optical components [4].

It is generally recognized that LID in polished optical surfaces is due to local defects. These potentially include distributed point defects, microinclusions, and microcracks generated during the polishing process. Optical-materialsurface processing inevitably modifies the near-surface layer as shown in Figure 1.

It is unlikely that large-aperture optical surfaces without any defects can be manufactured in the near future. Therefore, the useful lifetime of the optics depends largely on the rate at which damage can grow under subsequent illumination. The key point is full understanding of light interaction with the damaged material. 


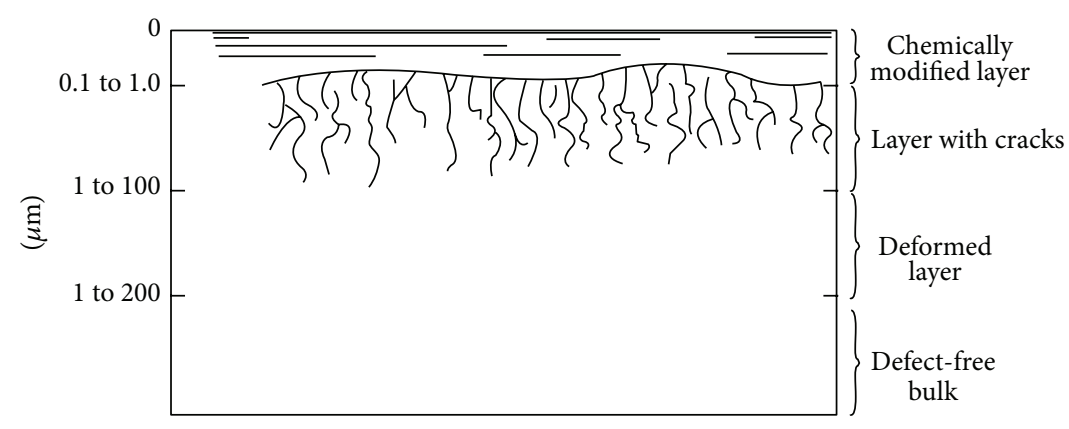

FIGURE 1: Schematic of the subsurface damage [5].

Several mechanisms are usually used to explain the laser absorption in the damage sites. Heating of fused silica can result in efficient absorption of UV radiation because of the loss of oxygen [6]. The high pressure [7], densified material, and structure changes $[8,9]$ generated by laser light absorption may initiate additional absorption. The interference of the scattered light caused by the crushed material and microcracks in damage spots produces local intensity spikes where breakdown can take place, igniting plasma and producing the enhanced absorption [10]. In reality, all of these mechanisms are probably important and manifest to some degree.

During last decades, numerous theoretical and experimental methods have been used to study the laser damage initiation and growth. The experimental studies focus on the test of laser-induced damage threshold, the characterization of damage morphology, and light modulation to the downstream optics. The theoretic studies focus on the mechanisms of laser damage initiation and growth, computational models to estimate damage behavior, and modulation of damage to laser beam quality. Development of damage mitigation techniques is currently a hot topic based on above researches.

In this paper, the damage mechanisms of optical materials initiated by laser pulse are firstly introduced. Thermal absorption and avalanche ionization of material during pulse irradiation are discussed in detail. Secondly, the recently reported experimental results about laser-induced damage are summarized. Finally, the typical theoretical models are described and some simulated results consistent with experiments are presented. In this review, we attempt to summarize the main mechanisms and related phenomena of laserinduced damage and provide reference for damage mitigation techniques.

\section{The Mechanisms of Laser-Induced Damage}

The effects of laser pulse duration on laser-induced damage in different materials are similar which points to properties of laser-induced damage which are material independent and give insight to the underlying physics of laser-induced damage [11]. It is clear that the investigations of damage mechanism are fundamental and essential.

There are generally two main mechanisms which cause laser-induced damage. The first one is thermal absorption, which arises from deposition of the laser energy in the material. This process may be generally observed during radiation of continuous wave, long pulse lengths, and highpulse-repetition-frequency pulse trains. The second one is electron avalanche, which arises when the electric field density is high enough or the energy is delivered at a high intensity to strip electrons from the lattice. These apply when the pulse lengths are short enough for avalanche ionization to take place and when the thermal absorption is low enough for the avalanche threshold to be below the thermal threshold. Laser-induced damage may be caused by single mechanism or both the above mechanisms in concert [12].

2.1. Thermal Absorption. Some energy of a laser incident on a material is absorbed in the form of heat. The interaction depends largely on the beam parameters, ambient conditions, component properties, and microstructure [13]. Meanwhile, most materials contain absorbing impurities which may originate either from the raw materials or from the manufacturing processes. The absorption is made up of two parts which are material intrinsic absorption and localized impurity absorption in the transmitting region of the spectrum.

2.1.1. Material Intrinsic Absorption. In a transmitting medium, laser radiation is absorbed in a cylinder passing through the material along the beam. It causes both local temperature rise of material and radial strain between the cylinder centre line and the edge of component. The temperature distribution and evolution as well as the radial strain depend on many factors, such as beam diameter, pulse duration, reflectivity, component size, thermal diffusion, and material strength. In order to avoid complications, appropriate simplification and assumptions are essential.

The power density deposited at a depth $z$ in a material with reflectivity $R$ by a laser with incident intensity $I(x, y, t)$ is given by [14-16]

$$
\begin{aligned}
J_{a}(x, y, t)= & I(x, y, t) \times(1-R) \alpha \\
& \times\left\{1-\exp \left[-\int_{0}^{z}\left(z^{\prime}\right) d z^{\prime}\right]\right\},
\end{aligned}
$$

where $\alpha$ is the absorption coefficient and $D$ is the thermal diffusivity of the material. The dimensions of $J_{a}$ are $J /\left(m^{3} s\right)$. 
It has been shown that thermal radiation from the hot surface can be neglected at all absorbed flux densities for pulses shorter than $10^{-6} \mathrm{~s}$ [14]. Moreover, if the thermal properties are considered independent of temperature, the differential equation for heat flow in a semi-infinite slab of material with a boundary at $z=0$ is [16]

$$
\begin{aligned}
\nabla^{2} T & (x, y, z, t)-\frac{1}{D} \frac{\partial T(x, y, z, t)}{\partial t} \\
& =\frac{J_{a}(x, y, z, t)}{\kappa},
\end{aligned}
$$

where $D$ is the thermal diffusivity of the slab and $\kappa$ is thermal conductivity.

If the centre of the beam reaches the melting point of the material under test, $T_{m}$, a damage threshold can be defined [12]:

$$
E_{D}=\frac{C d T}{\alpha},
$$

where $d T=T_{m}-T_{a}$ and $T_{a}$ is the ambient temperature, $C$ is the heat capacity of the component, and $\alpha$ is the absorption coefficient of the component.

A threshold for catastrophic cracking can similarly be defined [12]:

$$
E_{D}=\frac{C \kappa S}{\beta \alpha},
$$

where $S$ is the damaging stress and $\beta$ is the volume expansion coefficient.

2.1.2. Localized Impurity Absorption. Threshold is defined as the laser fluence when the temperature of matrix material with absorbers reaches the critical temperature $T_{c}$ due to local absorption induced by laser beam. A one-dimensional thermal diffusion model, considering heat conduction as the dominating energy-dissipation mechanism from the laserheated absorber, was first explored in the context of laser damage by Hopper and Uhlmann [17] and further developed by other authors [18-20]. According to the approximation of Feit and Rubenchik [20], assume (1) that absorber thermal conductivity is much higher than the conductivity of the matrix and (2) that the temperature is homogeneous inside the absorber. The temperature of the matrix surrounding the absorbing particle can be found from the heat equation [21]

$$
\frac{\partial T}{\partial t}=D \nabla^{2} T
$$

with boundary conditions at $r=a$ (particle radius)

$$
\alpha I(t)=-4 \kappa\left(\frac{\partial T}{\partial r}\right)_{r=a}+\frac{4}{3 \rho C a(\partial T / \partial r)_{r=a}},
$$

where $\alpha, \rho$, and $C$ are absorptivity, mass density, and heat capacity of the impurity particle, respectively; $D$ and $\kappa$ are thermal diffusivity and thermal conductivity of the matrix, respectively; and $I$ is laser intensity.
For the rectangular heating pulse of duration $\tau$, the solution of (5) is [21]

$$
T=T_{0}\left(1-\exp \left(-\frac{4 D \tau}{a^{2}}\right)\right)
$$

where $T_{0}=\alpha F a / 4 \kappa \tau, D=3 \kappa / 4 \rho C$, and $F$ is laser fluence; assuming $T=T_{c}$, the threshold fluence from (7) is

$$
F_{\text {th }}=6.3 T_{c} \kappa \sqrt{\frac{\tau}{\alpha}} \sqrt{D} .
$$

2.2. Electron Avalanche. In semiconductors and insulators, the generation of free carriers in the conduction band is the most notable effect induced by laser radiation. Under the conditions irradiated by high-power laser pulses, the carrier density can reach $10^{17}-10^{18} \mathrm{~cm}^{-3}$ which is called plasma.

At long wavelengths and for materials with narrow band gaps (e.g., semiconductors), thermal excitation is the dominant carrier generation mechanism [22].

Carriers are also generated by ionization across the band gap. If the photon energy of laser is larger than the band gap of material, carriers are generated very efficiently by a onephoton interband transition.

Transparent media are extremely large band gap materials in which photon energy is usually less than the band gap. Therefore, thermal ionization rarely occurs unless the temperature is extremely high. The mechanisms of free carrier generation existing in transparent materials are multiphoton interband transitions [23-26] and impact ionization [25-30].

2.2.1. Multiphoton Interband Transitions. In multiphoton interband transitions, $n$-photons are absorbed simultaneously and their total energy exceeds the band gap. The generalized transition rate for an $n$-photon process is written as [24]

$$
\frac{d N_{e}}{d t}=\frac{\sigma^{(n)} N_{0} I^{n}}{(h \omega)^{n}}
$$

where $\sigma^{(n)}$ is the cross-section for the $n$-photon process, $N_{0}$ is the ground-state population density, and $I$ is the local irradiance. Therefore, multiphoton ionization is a relevant mechanism of carrier generation only for small $n$ or for very intense (i.e., subpicosecond pulse-width) radiation [26].

2.2.2. Impact Ionization. Impact ionization occurs when a free carrier is accelerated at energy higher than the band gap by the electromagnetic field [31]. If a free carrier collides with a lattice atom, the energetic carrier is slowed down while another electron in the conduction band is excited and an electron avalanche is initiated. The equation that governs the generation rate of free carriers shows that the electron density grows exponentially with time [32]:

$$
\frac{\partial N_{e}}{\partial t}=N_{e} \frac{\partial}{\partial t}\left[\frac{\left\langle E_{e}\right\rangle}{E_{g}}\right]
$$

where $E_{e}$ is the energetic carrier kinetic energy and $E_{g}$ the band gap energy. 
TABLE 1: The relationships between the pulse length of high power and LID of components.

\begin{tabular}{l}
\hline Laser pulse length \\
Long pulse \\
Continuous wave \\
\hline Short pulse for ns level \\
\hline Extremely short pulse for ps level \\
\hline Ultrashort pulse for fs level \\
\hline \\
$\qquad 4 \%+200 \mathrm{~ms}$
\end{tabular}

(a)

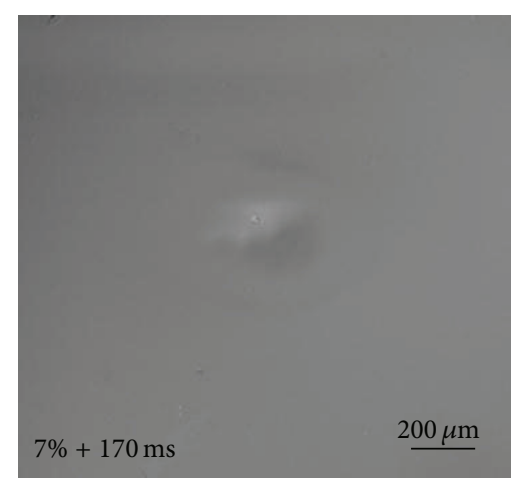

(b)
Damage phenomena

Stress, strain, melting, and ablation

Thermal absorption

Micropits, debris

Impact ionization Crater, plasma, and crush

Multiphoton-assisted impact ionization

Figure 2: Three typical surface morphologies observed in the laser irradiated areas of fused silica induced by $10.6 \mu \mathrm{m} \mathrm{CO}_{2}$ laser irradiation [37].

Traditional impact ionization holds that if the kinetic energy of a conduction band electron (CBE) is larger than the impact ionization threshold, the CBE can knock a valence band electron out of its band and promote it to the conduction band. It seems that traditional impact ionization can happen only if the kinetic energy of the CBE is larger than the threshold of the solid [33] and the avalanche coefficient of impact ionization is a constant with respect to laser intensity [34]. However, recent detailed experiments [35] show that the cold avalanche ionization (a CBE can knock out a new $\mathrm{CBE}$ even if the kinetic energy of that $\mathrm{CBE}$ is less than the threshold) exists and the avalanche coefficient changes with laser intensity.

Deng et al. presented a quantum theory of photonassisted impact ionization [36] to explain the cold avalanche ionization which shows that, in the presence of an intense laser, the impact ionization threshold is not unique and is coupled with the multiphoton process which leads to a lower threshold. Strictly speaking, under intense laser irradiation, the traditional impact ionization is replaced by photonassisted impact ionization. Theoretical and experimental research in this problem need further development.

2.3. Summary. In general, significant damage would not be observed for the transparent dielectric with a high band gap irradiated by low/medium power laser, and LID is only considered with intense radiation. Besides, mechanisms of laser-material interaction are dependent on the pulse length for the much shorter relaxation time of electrons than the lattice. The relationships between the pulse length of high-power laser and LID of components are summarized as shown in Table 1.

\section{Experimental Studies and Developments}

Materials are available for modern large optics in which the optical quality is high enough that bulk damage is either rare or completely absent compared to surface damage. Most of the observed damage is in the surface and near-surface of optics, which is associated with defects arising from the polishing process. What is more prevalent and dangerous is the rear (exit) surface damage. Therefore, mostly experiment researches focus on surface and subsurface damage.

3.1. Crater Morphology. Three typical surface morphologies (see Figure 2) of fused silica induced by $10.6 \mu \mathrm{m} \mathrm{CO}_{2}$ laser irradiation are observed [37], showing that obvious distortion does not occur for the material temperature below the softening point at low laser power; distortion occurs with the increase of laser power which means the material temperature is above the softening point; once the material temperature reaches the evaporation point, the surface distortion becomes clearer and a black ring appears around it, which has been identified as the debris being redeposited or recondensed from evaporated fused silica [38].

A damage morphology study performed with a $355 \mathrm{~nm}$, $8 \mathrm{~ns} \mathrm{Nd:YAG} \mathrm{laser} \mathrm{on} \mathrm{the} \mathrm{polished} \mathrm{fused} \mathrm{silica} \mathrm{sample} \mathrm{[39]}$ revealed that damage was initiated at micropits on both input and output surfaces and the micropits generated cracks on the surface at higher fluences.

A model system with artificial gold nanoparticles embedded inside the silica film deposited on a fused silica glass substrate was designed and two types of damage crater morphology were found in model systems [40-42]. For relatively shallow absorber locations, shown as a regular 


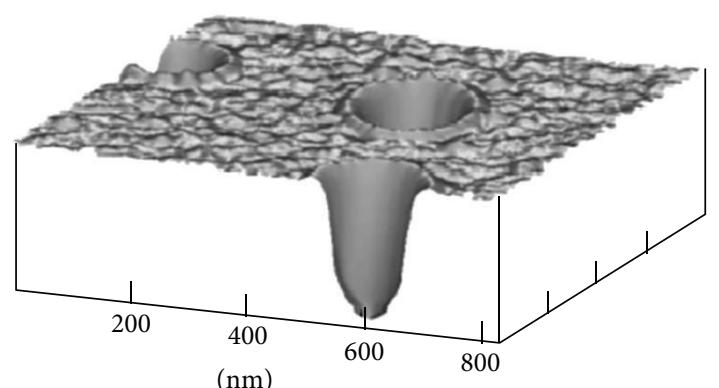

(a)

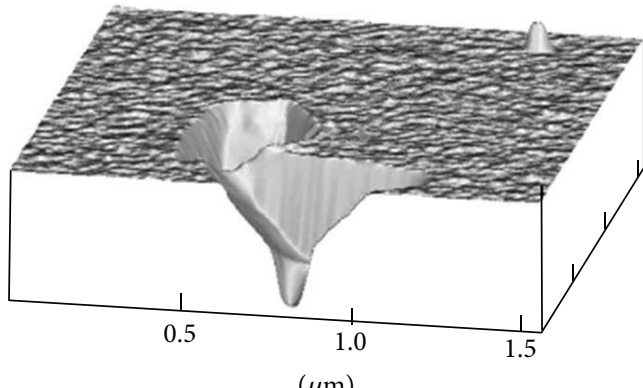

(b)

FIGURE 3: Characteristic crater morphologies produced by $351 \mathrm{~nm}, 0.5 \mathrm{~ns}$ irradiation of $18.5 \mathrm{~nm}$ particles lodged at different depths: (a) regular crater [40]; (b) complex crater [43].

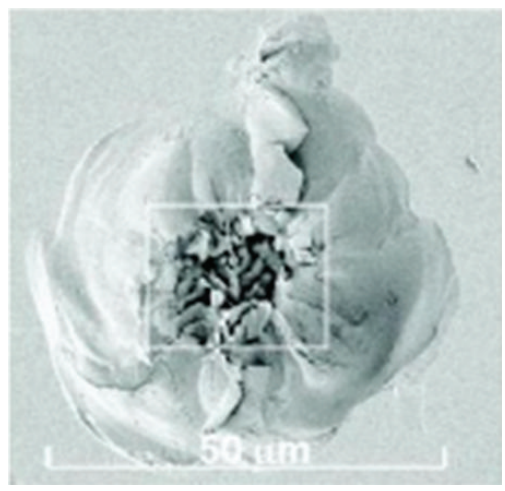

(a)

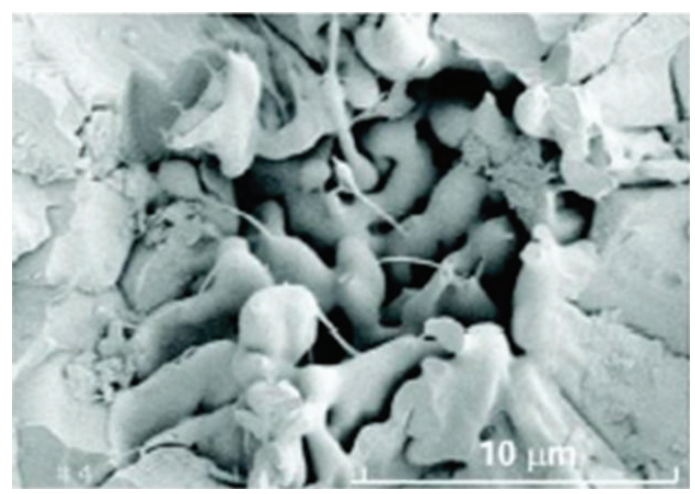

(b)

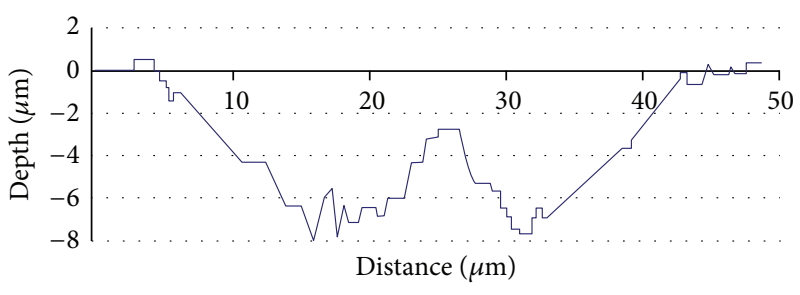

(c)

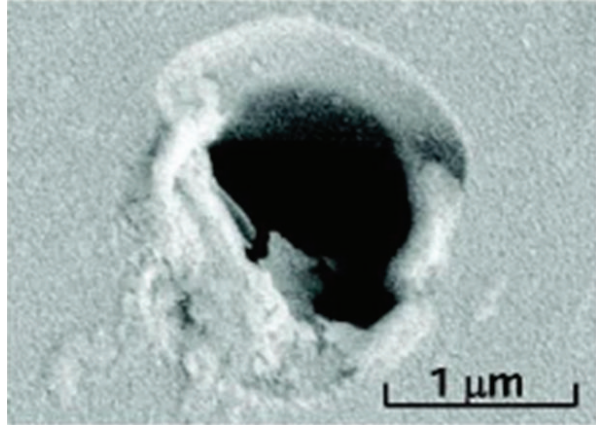

(d)

Figure 4: Damage craters produced in fused silica by $355 \mathrm{~nm}, 7.5 \mathrm{~ns}$ pulse with $45 \mathrm{~J} / \mathrm{cm}^{2}$ fluence: (a) large crater with melted central core and fractured periphery; (b) magnified image of the central core; (c) cross-sectional profile of the crater shown in (a); (d) small craters produced by melting and evaporation [44].

crater in Figure 3(a), the damage crater morphology is typical melting and evaporation and has circular lateral symmetry, single-cone shape, smooth walls, and an elevated rim due to melted material flow and resolidification. The second crater morphology (complex crater in Figure 3(b)), for deeper absorber locations, exhibits a double-cone cross-sectional profile, random lateral shape with sharp corners, and the absence of an elevated rim, all indicating that the upper portion of the crater is removed by fracture [43].

Surface-damage morphology of the fused silica glass finished according to fusion-scale laser specifications and irradiated by nanosecond pulses at $355 \mathrm{~nm}$ wavelength [44] reveals features similar to those observed in the model systems. A large crater shown in Figure 4(a) has a clearly melted core and periphery formed through fracture while a small crater shown in Figure 4(d) has features (symmetry, elevated rim) typical for explosive melting and evaporation.

The morphology of LID at optical materials has been discussed extensively. For high fluence $355 \mathrm{~nm}$ laser pulse, the damage craters consist of a molten core region with high density crater wall, surrounded by a near concentric region of 


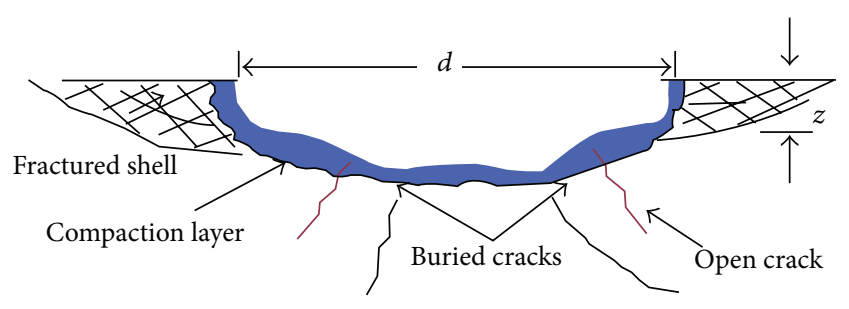

FIGURE 5: Schematics of damage crater morphology [44].

fractured material. The typical damage crater morphology is shown in Figure 5.

3.2. Damage Growth. When the laser fluence is continuously increased, damage starts to grow from the output surface into the bulk $[45,46]$. It shows damage depth and damage diameter in the output surface as a function of the number of shots. As observed by Pini et al. [47], there are two stages of damage growth: during stage I, both damage area and damage depth increase as a function of the number of shots; during stage II, only damage depth continues to grow.

The analysis of damage growth in fused silica induced by high fluence ultraviolet $355 \mathrm{~nm}$ laser pulses [44, 48] indicates that damage sites located at the entrance surface show linear growth with a number of pulses, in contrast to clearly exponential exit-surface-damage growth, and much deeper crack propagation appears in the case of exit surface compared to the entrance surface case. Morphology in the form of cracks is represented by a concentric fractured shell, where nonbridging oxygen hole centers are found, and by different sizes of (micro- and nanoscale) open and closed radial cracks $[44,48]$.

The experiments of damage growth on fused silica and DKDP samples found that, for higher laser fluences, the number and the emission intensity of the sites where plasma is formed increase while their size also expands [49]. The results strongly suggest that the absorbing modified layer as well as the mechanically modified material including cracks and crushed material can reignite the damage process.

3.3. Laser Intensity Modulation. Nonabsorbing defects, such as voids, microcracks, and local stress concentration, can serve as positive or negative lenses for the incident laser light [10] due to their refractive index different from the surrounding medium. The light intensity enhancement is dependent on the refractive index and geometry of the defects.

The typical morphology and downstream intensification effects associated with $\mathrm{CO}_{2}$ laser mitigation of fused silica are studied [50]. The results show that the intensification patterns are related to the elevated rims around the ablation pit. Control of the rims structure around typical mitigation sites is crucial in preventing damage to downstream optics.

Runkel et al. constructed a laser-based modulation measurement system which is capable of imaging any plane from 0 to $30 \mathrm{~cm}$ downstream from the defect, and modulation curves generated from these defects will be used to assess the risk of components to optical damage [51].

3.4. Summary. It is generally recognized that LID in components is initiated by the surface and subsurface absorbing defects. With the intense UV pulse laser, plasmas are emitted by thermal explosion of microabsorbers, which generates micropits and debris on the surface. During the subsequent irradiation, damage sites located at the entrance surface show linear growth with the number of pulses. However, exponential damage growth is observed at the exit surface where crush material and fracture propagation are common features. In addition, distortion, melting, and even ablation occur due to thermal effect. And an elevated rim probably appears around the damage site induced by flow and then resolidification of melted material. Defects and damages can lead to the electric field enhancement which reduced the LIDT.

\section{Theoretical Models and Numerical Analysis}

To accurately solve the laser damage threshold is very difficult when temperature dependence of thermal and optical properties, nonhomogeneous beam spatial profile, phase transformations of the heated material, and so forth are considered. Finding an analytical solution becomes impossible and numerical techniques need to be developed.

For the long pulse or continuous wave, ionization hardly occurs while thermal absorption is the dominant process. Temperature evolution, residual stress and strain, and melting morphology (if the laser intensity is high enough) can be estimated using heat conduction model [52].

In the ultrahigh-power, short-pulse laser irradiation, ionization and plasma generation is the dominant process. Damage crater formation is similar to crater formation due to meteorite impact or to underground explosion [53, 54]. First, thermal explosion indicates the process of plasma fireball generated by ionization. Then the laser absorption of plasma inside the material is released in different forms. Some energy drives crashed material movement (fireball growth model), some is transported by the shock (impact cratering model), and some is consumed in cracking (brittle-fracture model).

4.1. Thermal Diffusion Model. If only thermal absorption is concerned, the temperature of material and impurities increase. Thermal diffusion occurs by conduction and convection, and finally distortion, stress, melting, and even ablation appear in the material.

This process can be simulated by a finite-element method. We considered bulk fused silica with a surface heat source due to the small absorption depth of $\mathrm{CO}_{2}$ laser radiation [52]. The temperature evolution and residual stress distribution are estimated, which are consistent with experiments.

The reliability of simulated results is strongly dependent on the parameters of material, such as heat conductivity and yield strength, especially in high temperature where the accurate parameters are difficult to obtain. Elhadj et al. described an experimental and analytical approach to determine the 
temperature dependent intrinsic lattice thermal conductivity by comparing the experimental data with the calculated data [55]. The thermal conductivity as a function of temperature based on this method shows good agreement with reported one.

4.2. Thermal Explosion and Fireball Growth Model. The size of damage craters observed at the front and rear surface can be estimated using the thermal explosion theory [56]. According to this theory, reaching critical temperature in the vicinity of the absorbing defect leads to the effective conversion of the defect-surrounding matrix to the absorbing medium, rapid heating, and ionization (thermal explosion). The result is a plasma "fire-ball" formation.

Laser damage growth at optical surface is strongly influenced by laser-plasma interactions. Laser-generated plasma always expands towards the beam source [32] which means that at the rear surface the free carrier plasma expands inside the material while at the front surface it expands in the atmosphere. Under subsequent laser shots, energy deposition at the front surface is reduced by the shielding effect of the plasma. For the rear surface, damage growth occurs by energy deposition into plasma inside the material (Figure 6). As a result, LID at rear surface of components is more severe.

For the rear surface, the material inside which plasma expands can be heated and ionized by laser-plasma interactions and damage growth occurs, which can be estimated using plasma fireball growth model.

Generation and growth of plasma fireball can be treated as the hydrodynamic motion of the highly heated and crashed material leading to crater formation. The explosion is considered as instantaneous energy deposition and initiates material motion ina simple hydrodynamic description of crater formation [57], which agrees well with the experimental data and verifies the validity of the hypothesis of damage initiation by small absorbers.

4.3. Impact Cratering Model. Plasma at rear surface of components ejects into the material with certain initial kinetic energy and then the energy is redistributed between plasma and material. Under subsequent intense irradiation, a strong shock wave is launched in both plasma and material along their interface. The resulting pressure vastly exceeds the strength of material and the plasma penetrates the interface which leads to component deformation, material crash, and crater deformation.

A rough estimate of the partition of the initial kinetic energy of the projectile can be made with the planar impact approximation [58]. In this approximation, the plasma is modeled as a sheet of material with specified thickness and diameter which is launched with some velocity on a flat halfspace of target material [59]. Shock waves develop in the plasma and the material can be demonstrated, respectively, and impact crater size can be estimated.

4.4. Brittle-Fracture Model. The experiments on damage growth carried out at Lawrence Livermore National Laboratory (LLNL) revealed a reproducible and consistent pattern

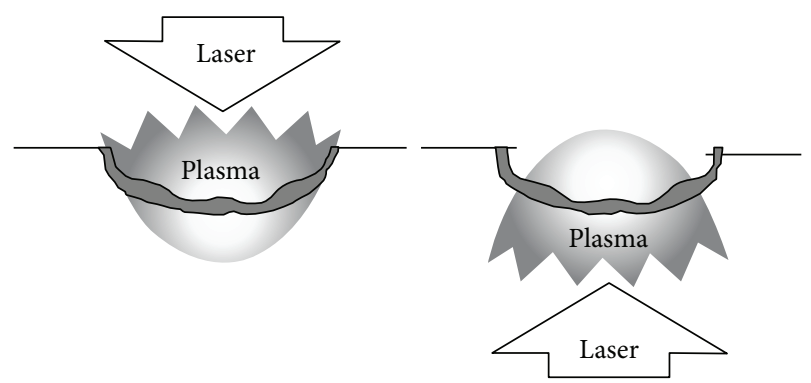

FIgURE 6: Schematic illustration of the damage-plasma interaction with surface material during front and rear surface irradiation [21].

of damage growth [60]: the transverse size of damage sites increases with the number of pulses in an accelerated way, and the site size grows mainly by the development of conical cracks on the periphery of the damage spot.

Cracks may occur when the stress (especially tensile stress for the lower tensile strength comparing with the compressive strength of most materials) in brittle material exceeds the strength. Two approaches produce stress during laser pulse. One is thermal stress arising from thermal expansion of heating material and the other is mechanical stress caused by shock wave after explosion. In the first case, the microabsorber on the optical surface can be heated rapidly when irradiated by a long pulse length and highpower laser. Compressive stress in the surrounding material is generated by the thermal expansion of absorber during the heating process and then converted into tensile stress during the cooling process which can cause cracks in the brittle material. The strong dependence between the crack propagation and the cooling rate observed in experiments [61] exactly indicates that fracture is caused by tensile stress during cooling process. However, a short-pulse length and high-power laser is suitable for the second case. A strong shock wave generated by thermal explosion of microabsorber located in the rear surface is launched into the material which can lead to deformation and crush of material. When the shock wave reaches the free surface and reflects back, tensile stress is generated and easily initiates cracks.

It is noticed that fracture usually does not occur in the thermal diffusion model. Plastic deformation rather than fracture occurs due to reduced strength and increased viscosity when the material temperature arises.

Numerical finite-element calculations using brittlefracture models can predict the kinetics of propagation and final shape of the crack [62]: a cone-shaped crater after crashed material removal. Another example is numerical modeling of crack-propagation kinetics [63]: a 1D hydrodynamic code capable of computing laserenergy deposition is used as the preprocessor, and a 2D finite-element hydrodynamic code predicts fracture evolution which shows that crack propagation for the chosen irradiation parameters has speed of the order of $\sim 1 \mathrm{~km} / \mathrm{s}$, comparable to the speed of sound.

4.5. Electric Field Enhancement Model. The electric field enhancement, also known as light intensity modulation, 
TABLE 2: Theoretical models of laser-induced damage.

\begin{tabular}{lll}
\hline Theoretical models & Damage mechanisms & Applications \\
\hline Thermal diffusion & Intrinsic absorption & Stress, strain, melting, and ablation \\
\hline Thermal explosion & Electron avalanche & Damage crater initiation \\
\hline Fireball growth & Electron avalanche & Damage growth on rear surface \\
\hline Impact cratering & Shock wave due to explosion & Damage growth on rear surface \\
\hline Brittle fracture & Impurity absorption & Cracks generation and propagation \\
\hline
\end{tabular}

exists in two forms during a light beam as an electromagnetic wave through optical materials, that is, nonlinear self-focusing and constructive interference. Nonlinear selffocusing arises from heterogeneous refractive index of material. Temperature rise, stress, and distortion due to laser radiation can change the refractive index of material and make it act like a focusing lens which leads to the electric field enhancement. A similar situation also occurs for defects and impurities in the material. Furthermore, the interference between incident and reflecting light at the interface of different media, such as material/impurity interface and material/air interface, can extremely enhance the electric field. The significant modulations of laser intensity due to damage craters on the optical surface are detected in the experiments $[10,50]$ which remarkably reduce the damage threshold.

The process of laser radiation can be considered as the propagation of an electromagnetic wave through material, which satisfies the Maxwell equations. The 3D finite difference time domain (FDTD) method can be used to solve the equations to obtain the electric field intensity distribution. A study on laser modulation by surface micro-defects on $\mathrm{KH}_{2} \mathrm{PO}_{4}$ crystal [64] shows that the light intensity distribution inside the crystal is badly distorted and modulation properties are strongly dependent on the defects geometries. The effects of contamination coating thickness in fused silica rear surface on the UV laser intensity modulation are discussed [65], which reveal that light intensity enhancement factor (LIEF) increases with the contamination thickness, and the modulation becomes more remarkable with time for the raise of surface temperature. In [66], the modulation to incident laser by a Gaussian damage crater on the front surface of fused silica leads to the annular laser enhancement on the rear surface which is consistent with the experiments.

4.6. Summary. Self-consistent, comprehensive modeling of laser damage initiation and growth must include various physical effects. From the aspect of material, these effects include plasma generation, the laser interaction with plasma fireball, fireball expansion, cracks formation and development, phase transformations in surrounding materials, and the flow of crushed material. From the other aspects of laser beam, the physical effects may include scattering, interference, birefringence, self-focusing, and harmonic generation. The existent modeling includes only a part of these physics $[62,63]$. The more important thing is that the two aspects are considered independently, which are actually coupled with each other. Nevertheless, these models are able to catch some essential features of damage morphology. A simple summary of these laser damage models is shown in Table 2.

\section{Conclusion}

It is impossible to prevent the optical components in largeaperture laser systems from laser-induced damage at present. Even initially perfect optics could be damaged due to mishandling or contamination during operation. The methods of damage growth mitigation must be developed to remove the modified material from the damage spot and heal the cracks.

Recently developed techniques for surface laser conditioning, contamination removal, and damage mitigation, such as wet chemical etching, cold plasma etching, $\mathrm{CO}_{2}$ laser processing, and microflame torch processing [67], make it possible to significantly extend the lifetime of optical components in high-power laser systems.

Further challenges, related to ever-increasing laser-power densities, can be met only by means of better understanding of surface-damage mechanisms. Progress in detailed theoretical modeling of extrinsically and intrinsically driven damage is of crucial importance. In addition, calculation of multiphysics coupling should be developed especially between the defects or the modified materials and laser modulation.

\section{Conflict of Interests}

The authors declare that there is no conflict of interests regarding the publication of this paper.

\section{Acknowledgments}

This work is financially supported by the National Natural Science Foundation of China (Grant no. 61178018) and the Ph.D. Funding Support Program of Education Ministry of China (Grant no. 20110185110007).

\section{References}

[1] F. Y. Génin, K. Michlitsch, J. Furr, M. R. Kozlowski, and P. A. Krulevitch, "Laser-induced damage of fused silica at 355 and $1064 \mathrm{~nm}$ initiated at aluminum contamination particles on the surface," in Laser-Induced Damage in Optical Materials, Proceedings of SPIE, pp. 126-138, May 1997. 
[2] F. Y. Genin, A. M. Rubenchick, A. K. Burnham et al., "Thin film contamination effects on laser-induced damage of fused silica surfaces at $355 \mathrm{~nm}$," in 3rd International Conference on Solid State Lasers for Application to Inertial Confinement Fusion, Proceedings of SPIE, pp. 212-218, Monterey, Calif, USA, June 1998.

[3] F. Y. Génin, M. D. Feit, M. R. Kozlowski, A. M. Rubenchik, A. Salleo, and J. Yoshiyama, "Rear-surface laser damage on 355$\mathrm{nm}$ silica optics owing to Fresnel diffraction on front-surface contamination particles," Applied Optics, vol. 39, no. 21, pp. 3654-3663, 2000.

[4] J. H. Campbell, P. A. Hurst, D. D. Heggins, W. A. Steele, and S. E. Bumpas, "Laser induced damage and fracture in fused silica vacuum windows," in Laser-Induced Damage in Optical Materials, Proceedings of SPIE, October 1996.

[5] P. E. Miller, T. I. Suratwala, L. L. Wong et al., "The distribution of subsurface damage in fused silica," in Proceedings of the 37th Annual Boulder Damage Symposium: Annual Symposium on Optical Materials for High Power Lasers, Boulder, Colo, USA, September 2005.

[6] A. K. Burnham, M. Runkel, S. G. Demos, M. R. Kozlowski, and P. J. Wegner, "Effect of vacuum on the occurrence of UV-induced surface photoluminescence, transmission loss, and catastrophic surface damage," in Proceedings of the International Symposium on Optical Science and Technology, pp. 243-252, August 2000.

[7] N. Bourne, J. Millett, and J. Field, "On the strength of shocked glasses," Proceedings of the Royal Society of London A: Mathematical, Physical and Engineering Sciences, vol. 455, no. 1984, pp. 1275-1282, 1999.

[8] J. Wong, D. Haupt, J. H. Kinney et al., "Nature of damage in fused silica induced by high-fluence 3-omega 355-nm laser pulses, a multiscale morphology microstructure, and defect chemistry study," in Laser-Induced Damage in Optical Materials, vol. 4347 of Proceedings of SPIE, pp. 466-467, 2001.

[9] A. Kubota, M.-J. Caturla, L. Davila et al., "Structural modifications in fused silica due to laser-damage-induced shock compression," in Laser-Induced Damage in Optical Materials, vol. 4679 of Proceedings of SPIE, Boulder, Colo, USA, October 2001.

[10] M. D. Feit and A. M. Rubenchik, "Laser intensity modulation by nonabsorbing defects," in Proceedings of the 2nd International Conference on Solid State Lasers for Application to ICF, pp. 475480, 1997.

[11] C. W. Carr, M. J. Matthews, J. D. Bude, and M. L. Spaeth, "The effect of laser pulse duration on laser-induced damage in $\mathrm{KDP}$ and $\mathrm{SiO}_{2}$," in Proceeding of the 37th Annual Boulder Damage Symposium: Laser-Induced Damage in Optical Materials, September 2006.

[12] R. M. Wood, Laser-Induced Damage of Optical Materials, CRC Press, New York, NY, USA, 2003.

[13] X. Chen, X. Zu, W. Zheng et al., "Experimental research of laserinduced damage mechanism of the sol-gel $\mathrm{SiO}_{2}$ and ibsd $\mathrm{SiO}_{2}$ thin films," Acta Physica Sinica, vol. 55, no. 3, pp. 1201-1206, 2006.

[14] J. Ready, Effects of High-Power Laser Radiation, Elsevier, New York, NY, USA, 2012.

[15] M. Ohring, Materials Science of Thin Films, Academic Press, New York, NY, USA, 2001.

[16] H. S. Carslaw and J. C. Jaeger, Conduction of Heat in Solids, 1959.
[17] R. Hopper and D. R. Uhlmann, "Mechanism of inclusion damage in laser glass," Journal of Applied Physics, vol. 41, no. 10, pp. 4023-4037, 1970.

[18] T. W. Walker, A. H. Guenther, and P. Nielsen, "Pulsed laserinduced damage to thin-film optical coatings-Part II: theory," IEEE Journal of Quantum Electronics, vol. 17, no. 10, pp. 2053$2065,1981$.

[19] M. Fuka and J. McIver, "Effects of thermal conductivity and index of refraction variation on the inclusion dominated model of laser-induced damage," Proceedings of SPIE, the International Society for Optical Engineering, vol. 1438, pp. 576-583, 1990.

[20] M. D. Feit and A. M. Rubenchik, "Implications of nanoabsorber initiators for damage probability curves, pulselength scaling and laser conditioning," in Laser-Induced Damage in Optical Materials, vol. 5273 of Proceedings of SPIE, September 2003.

[21] S. Papernov and A. W. Schmid, "Laser-induced surface damage of optical materials: Absorption sources, initiation, growth, and mitigation," in Proceedings of the 40th Annual Boulder Damage Symposium-Laser-Induced Damage in Optical Materials, September 2008.

[22] I. W. Boyd, T. D. Binnie, J. I. B. Wilson, and M. J. Colles, "Absorption of infrared radiation in silicon," Journal of Applied Physics, vol. 55, no. 8, pp. 3061-3063, 1984.

[23] P. Audebert, P. Daguzan, A. dos Santos et al., "Space-time observation of an electron gas in $\mathrm{SiO}_{2}$," Physical Review Letters, vol. 73, no. 14, pp. 1990-1993, 1994.

[24] W. W. Duley, UV Lasers: Effects and Applications in Materials Science, Cambridge University Press, New York, NY, USA, 2005.

[25] S. C. Jones, P. Braunlich, R. T. Casper, X.-A. Shen, and P. Kelly, "Recent progress on laser-induced modifications and intrinsic bulk damage of wide-gap optical materials," Optical Engineering, vol. 28, no. 10, Article ID 281039, 1989.

[26] B. C. Stuart, M. D. Feit, A. M. Rubenchik, B. W. Shore, and M. D. Perry, "Laser-induced damage in dielectrics with nanosecond to subpicosecond pulses," Physical Review Letters, vol. 74, no. 12, pp. 2248-2251, 1995.

[27] M. Bass and H. H. Barrett, "Avalanche breakdown and the probabilistic nature of laser-induced damage," Quantum Electronics, vol. 8, no. 3, pp. 338-343, 1972.

[28] N. Bloembergen, "Laser-induced electric breakdown in solids," IEEE Journal of Quantum Electronics, vol. 10, no. 3, pp. 375-386, 1974.

[29] D. W. Fradin, E. Yablonovitch, and M. Bass, "Confirmation of an electron avalanche causing laser-induced bulk damage at 1.06 $\mu \mathrm{m}$," Applied Optics, vol. 12, no. 4, pp. 700-709, 1973.

[30] W. L. Smith, J. H. Bechtel, and N. Bloembergen, "Picosecond laser-induced damage morphology: spatially resolved microscopic plasma sites," Optics Communications, vol. 18, no. 4, pp. 592-596, 1976.

[31] F. Seitz, "On the theory of electron multiplication in crystals," Physical Review, vol. 76, no. 9, pp. 1376-1393, 1949.

[32] M. von Allmen and A. Blatter, Laser-Beam Interactions with Materials: Physical Principles and Applications, Springer, Berlin, Germany, 1995.

[33] B. K. Ridley, Quantum Processes in Semiconductors, Oxford University Press, New York, NY, USA, 2013.

[34] B. C. Stuart, M. D. Feit, S. Herman, A. M. Rubenchik, B. W. Shore, and M. D. Perry, "Nanosecond-to-femtosecond laserinduced breakdown in dielectrics," Physical Review B, vol. 53, no. 4, article 1749, 1996. 
[35] P. P. Rajeev, M. Gertsvolf, P. B. Corkum, and D. M. Rayner, "Field dependent avalanche ionization rates in dielectrics," Physical Review Letters, vol. 102, no. 8, Article ID 083001, 2009.

[36] H. X. Deng, X. T. Zu, X. Xiang, and K. Sun, "Quantum theory for cold avalanche ionization in solids," Physical Review Letters, vol. 105, no. 11, Article ID 113603, 2010.

[37] X. Xia, Z. Wan-Guo, Y. Xiao-Dong et al., "Irradiation effects of $\mathrm{CO}_{2}$ laser parameters on surface morphology of fused silica," Chinese Physics B, vol. 20, no. 4, Article ID 044208, 2011.

[38] I. L. Bass, G. M. Guss, and R. P. Hackel, "Mitigation of laser damage growth in fused silica with a galvanometer scanned $\mathrm{CO}_{2}$ laser," in Laser-Induced Damage in Optical Materials, vol. 5991 of Proceedings of SPIE, Boulder, Colo, USA, September 2005.

[39] J. Yoshiyama, F. Y. Genin, A. Salleo et al., "Effects of polishing, etching, cleaving, and water leaching on the UV laser damage of fused silica," in Laser-Induced Damage in Optical Materials: 1997, vol. 3244 of Proceedings of SPIE, Boulder, Colo, USA, October 1997.

[40] S. Papernov and A. W. Schmid, "Two mechanisms of crater formation in ultraviolet-pulsed-laser irradiated $\mathrm{SiO}_{2}$ thin films with artificial defects," Journal of Applied Physics, vol. 97, no. 11, Article ID 114906, 2005.

[41] S. Papernov and A. W. Schmid, "Localized absorption effects during $351 \mathrm{~nm}$, pulsed laser irradiation of dielectric multilayer thin films," Journal of Applied Physics, vol. 82, no. 11, pp. 54225432, 1997.

[42] J. DiJon, T. Poiroux, and C. Desrumaux, "Nano absorbing centers: a key point in the laser damage of thin films," in LaserInduced Damage in Optical Materials, vol. 2966 of Proceedings of SPIE, 1997.

[43] S. I. Kudryashov, S. D. Allen, S. Papernov, and A. W. Schmid, "Nanoscale laser-induced spallation in $\mathrm{SiO}_{2}$ films containing gold nanoparticles," Applied Physics B: Lasers and Optics, vol. 82, no. 4, pp. 523-527, 2006.

[44] J. Wong, J. L. Ferriera, E. F. Lindsey, D. L. Haupt, I. D. Hutcheon, and J. H. Kinney, "Morphology and microstructure in fused silica induced by high fluence ultraviolet $3 \omega(355 \mathrm{~nm})$ laser pulses," Journal of Non-Crystalline Solids, vol. 352, no. 3, pp. 255272, 2006.

[45] A. Salleo, R. Chinsio, and F. Y. Genin, "Crack propagation in fused silica during UV and IR nanosecond-laser illumination," in Laser-Induced Damage in Optical Materials: 1998, vol. 3578 of Proceedings of SPIE, Boulder, Colo, USA, September 1998.

[46] A. Salleo, T. Sands, and F. Y. Génin, "Machining of transparent materials using an IR and UV nanosecond pulsed laser," Applied Physics A, vol. 71, no. 6, pp. 601-608, 2000.

[47] R. Pini, R. Salimbeni, G. Toci, and M. Vannini, "High-quality drilling with copper vapour lasers," Optical and Quantum Electronics, vol. 27, no. 12, pp. 1243-1256, 1995.

[48] M. A. Stevens-Kalceff, A. Stesmans, and J. Wong, "Defects induced in fused silica by high fluence ultraviolet laser pulses at 355 nm," Applied Physics Letters, vol. 80, article 758, no. 5, 2002.

[49] S. G. Demos, M. R. Kozlowski, M. Staggs, L. L. Chase, A. Burnham, and H. B. Radousky, "Mechanisms to explain damage growth in optical materials," in Proceedings of the 32nd Annual Boulder Damage Symposium - Laser-Induced Damaged in Optical Materials, pp. 277-284, 2001.

[50] M. J. Matthews, I. L. Bass, G. M. Guss, C. C. Widmayer, and F. L. Ravizza, "Downstream intensification effects associated with $\mathrm{CO}_{2}$ laser mitigation of fused silica," in Laser-Induced Damage in Optical Materials: 2007, vol. 6720 of Proceedings of SPIE, Boulder, Colo, USA, September 2007.

[51] M. Runkel, R. Hawley-Fedder, C. Widmayer, W. Williams, C. Weinzapfel, and D. Roberts, "A system for measuring defect induced beam modulation on inertial confinement fusion-class laser optics," in Proceedings of the Laser-Induced Damage in Optical Materials, Boulder, Colo, USA, September 2005.

[52] J. Yu, S. He, X. Xiang et al., "High temperature thermal behaviour modeling of large-scale fused silica optics for laser facility," Chinese Physics B, vol. 21, no. 6, Article ID 064401, 2012.

[53] M. Nordyke, "An analysis of cratering data from desert alluvium," Journal of Geophysical Research, vol. 67, no. 5, pp. 19651974, 1962.

[54] H. J. Melosh, "Impact ejection, spallation, and the origin of meteorites," Icarus, vol. 59, no. 2, pp. 234-260, 1984.

[55] S. Elhadj, M. J. Matthews, S. T. Yang et al., "Determination of the intrinsic temperature dependent thermal conductivity from analysis of surface temperature of laser irradiated materials," Applied Physics Letters, vol. 96, no. 7, Article ID 071110, 2010.

[56] Y. K. Danileikko, A. A. Manenkov, and V. Nechitaĭlo, "The mechanism of laser-induced damage in transparent materials, caused by thermal explosion of absorbing inhomogeneities," Soviet Journal of Quantum Electronics, vol. 8, no. 1, p. 116, 1978.

[57] M. Feit, L. Hrubesh, A. Rubenchik, and J. Wong, "Scaling relations for laser damage initiation craters," in Laser-Induced Damage in Optical Materials, vol. 4347 of Proceedings of SPIE, 2001.

[58] H. J. Melosh, Impact Cratering: A Geologic Process, vol. 1, Oxford University Press, New York, NY, USA, 1989.

[59] A. Salleo, "High-power laser damage in fused silica," ProQuest Information and Learning, 2001.

[60] M. A. Norton, L. W. Hrubesh, Z. Wu et al., "Growth of laser initiated damage in fused silica at $351 \mathrm{~nm}$," in Laser-Induced Damage in Optical Materials, vol. 4347 of Proceedings of SPIE, p. $468,2001$.

[61] M. Matthews, J. Stolken, R. Vignes et al., "Residual stress and damage-induced critical fracture on $\mathrm{CO}_{2}$ laser treated fused silica," in Laser-Induced Damage in Optical Materials, vol. 7504 of Proceedings of SPIE, December 2009.

[62] M. D. Feit, J. H. Campbell, D. R. Faux et al., "Modeling of laserinduced surface cracks in silica at $355 \mathrm{~nm}$," in Laser-Induced Damage in Optical Materials: 1997, vol. 3244 of Proceedings of SPIE, Boulder, Colo, USA, October 1997.

[63] F. Bonneau, P. Combis, J. Vierne, and G. Daval, "Simulations of laser damage of $\mathrm{SiO}_{2}$ induced by a spherical inclusion," in 32nd Annual Boulder Damage Symposium-Laser-Induced Damaged in Optical Materials, 2001.

[64] C. Ming-Jun, C. Jian, L. Ming-Quan, and X. Yong, "Study of modulation property to incident laser by surface micro-defects on $\mathrm{KH}_{2} \mathrm{PO}_{4}$ crystal," Chinese Physics B, vol. 21, no. 6, Article ID $064212,2012$.

[65] L. Li, X. Xiang, X.-T. Zu et al., "Modulation of incident laser by the defect site with a contamination coating on fused silica surface," Optik, vol. 124, no. 13, pp. 1637-1640, 2013.

[66] L. Li, X. Xia, Z. Xiao-Tao et al., "Numerical simulation of the modulation to incident laser by the repaired damage site in a fused silica subsurface," Chinese Physics B, vol. 20, no. 7, Article ID 074209, 2011.

[67] L. W. Hrubesh, M. A. Norton, W. A. Molander et al., "Methods for mitigating surface damage growth in NIF final optics," in Laser-Induced Damage in Optical Materials, Proceedings of SPIE, 2002. 

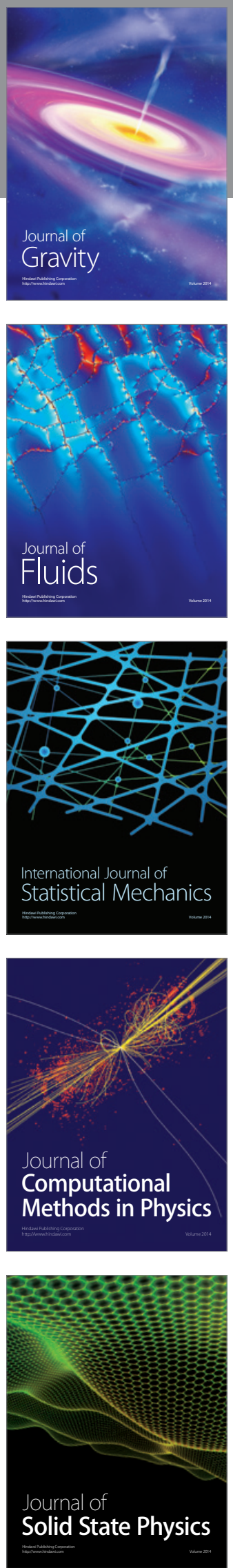

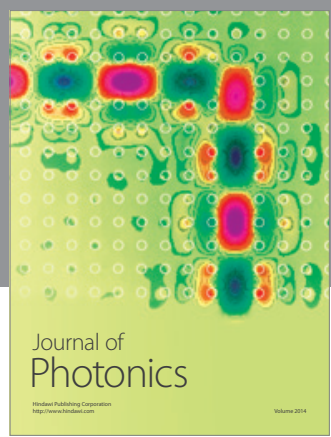

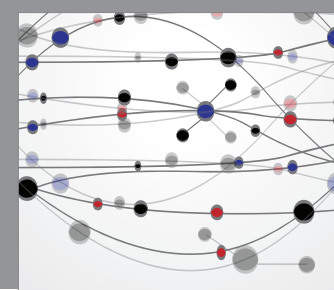

The Scientific World Journal

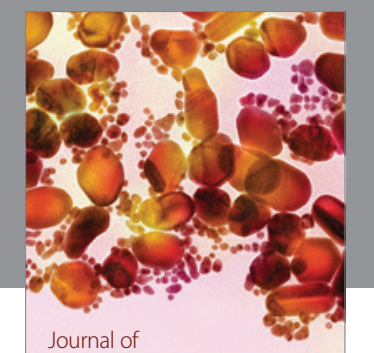

Soft Matter
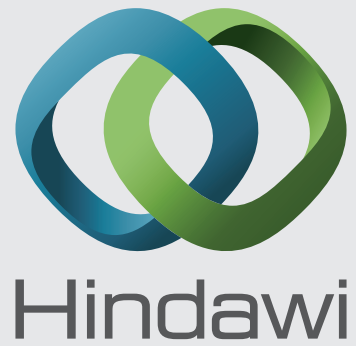

Submit your manuscripts at

http://www.hindawi.com
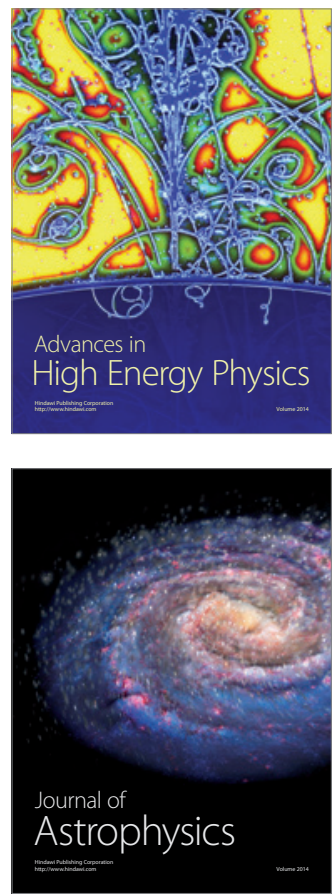
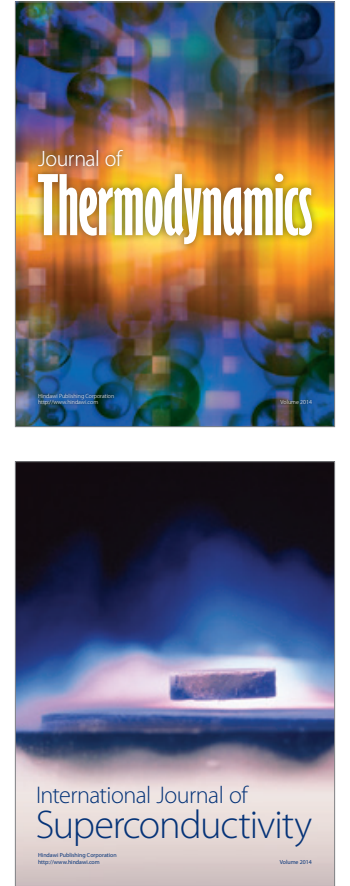
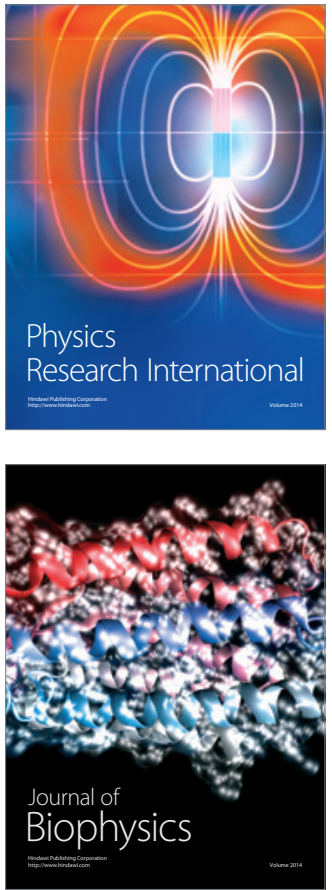
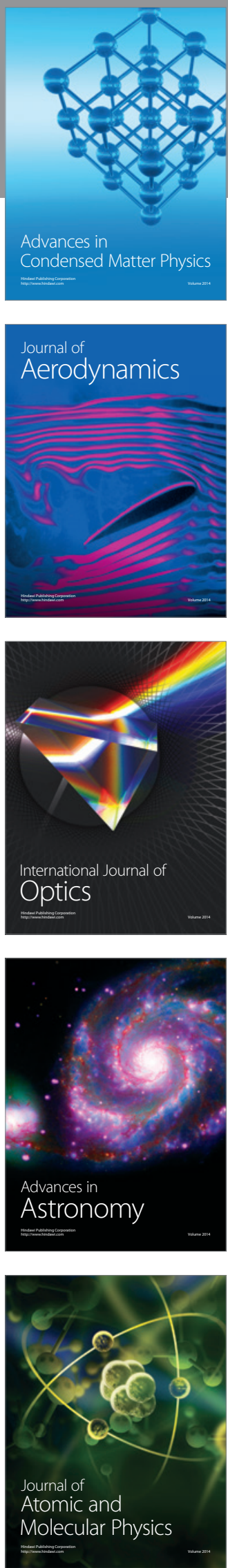